\title{
The Use of ATR-FTIR Spectroscopy for Quantification of Adsorbed Compounds
}

\author{
Giora Rytwo, ${ }^{1,2}$ Roee Zakai, ${ }^{1}$ and Bernd Wicklein ${ }^{3}$ \\ ${ }^{1}$ Department of Environmental Sciences, Tel Hai College, 12210 Upper Galilee, Israel \\ ${ }^{2}$ Environmental Physical Chemistry Laboratory, Galilee Research Center (MIGAL), 11016 Kiryat Shmona, Israel \\ ${ }^{3}$ Materials Science Institute of Madrid, Sor Juana Inés de la Cruz 3, 28049 Madrid, Spain \\ Correspondence should be addressed to Giora Rytwo; rytwo@telhai.ac.il
}

Received 22 October 2014; Accepted 6 February 2015

Academic Editor: Feride Severcan

Copyright (C) 2015 Giora Rytwo et al. This is an open access article distributed under the Creative Commons Attribution License, which permits unrestricted use, distribution, and reproduction in any medium, provided the original work is properly cited.

\begin{abstract}
Quantification of adsorbed amounts requires in most cases several assumptions. Adsorption of organic compounds, for example, is usually measured indirectly, by mass balance calculations based on the evaluation of the remaining chemical in solution. Such procedure might yield overestimates when precipitation or degradation of the adsorbate occurs and underestimates when separation of the sorbent material (e.g., clay particles) with the adsorbed chemical is not effective. This study presents a simple quantification procedure based on the ratio between IR absorption bands of the sorbate and the adsorbate. The advantages of the procedure are (a) direct evaluation of the adsorbed amount and (b) accurate measurement of chemicals that are hard to quantify, as those that do not absorb light in the UV-Visible range, or require expensive chromatography procedures.
\end{abstract}

\section{Introduction}

Quantification of adsorbed amounts of compounds on sorbates in general and clays in particular is usually performed by evaluating mass balance based on chromatography [1], UV-Visible spectroscopy [2,3], elemental analysis [4], or any other analytical technique that measures the remaining concentration of the analyte in solution. The general procedure when using those techniques is that if the added concentration is known $\left(\mathrm{C}_{\text {added }}\right)$ and the remaining and notadsorbed equilibrium concentration $\left(\mathrm{C}_{\text {equ }}\right)$ is measured by one of those analytical procedures, the adsorbed amount can be calculated by subtracting $\left(\mathrm{C}_{\text {added }}-\mathrm{C}_{\text {equ }}\right)$ and dividing it by the amount of sorbent.

One of the problems of such techniques is that several other processes that reduce concentration in equilibrium solution might be wrongly ascribed as adsorption. For example, Rytwo and coworkers [5] described apparent "adsorption" of crystal violet on Texas vermiculite that after a more detailed analysis was revealed as degradation of the dye on the surface of the mineral, and the amount adsorbed as crystal violet was considerably lower than the initial estimations. Overestimated adsorbed amounts may also stem from precipitation or evaporation of the adsorbate in case. Such erroneous quantifications might be avoided by measuring the adsorbate content not in the solution but directly on the sorbate. An example of such technique is the use of CHNSO analyzer that might yield the amount of organic compounds adsorbed on a mineral $[3,6]$ : considering the sorbent in most cases does not include carbon, nitrogen, or sulfur, all the amounts of those elements measured are ascribed to the organic compound adsorbed. In cases where the sorbent contains some of those elements more cumbersome evaluations can be performed to take that into consideration [7]. However, large discrepancies between different techniques are in some cases measured: Aznar and coworkers [3] had shown a 20\% difference between elemental analysis of the adsorbed amount and mass balance by UVVis. Elemental analysis will also not detect degradation on the surface, as disclosed by the study mentioned above [5], if the products remain bound to the sorbate.

Vibrational spectrum of a compound is considered to be a unique characteristic of the molecule; thus, infrared (IR) spectroscopy is used as a fingerprint technique for 
identification [8]. It has been used for decades in clay mineralogy to derive information concerning clay minerals structure, composition, and structural changes upon chemical modification [9]. It is an economical, nondestructive, rapid, and common technique that helps in mineral identification and give also unique information about clay minerals [10], including quantitative mineral analysis and composition of sediments and clays [11, 12], wetting/drying changes and their influences $[13,14]$, clay-organic interactions $[7,15]$, and even orientation of organic molecules on the mineral $[16,17]$. The increased sensitivity of Fourier transform infrared (FTIR) spectrometers makes the attenuated total reflectance (ATR) method a simple and routine technique. ATR has been used extensively to investigate adsorption of organic substances on minerals. Its main advantage is that it allows measurement of dispersions, gels, liquids, and pastes, very fast, with no extra preparation procedures [10].

This study reports an ATR-FTIR quantification technique performed on the sorbate and based on the quantitative evaluation of the ratio between adsorption bands of the adsorbate (in several cases an organic compound) and the sorbate (in the examples presented in this study a clay mineral). The technique is compared with results obtained by other analytical procedures such as gravimetry, UV-Visible spectroscopy, and CHN elemental analysis.

\section{Materials and Methods}

2.1. Materials. Volclay KWK Food Grade bentonite clay (American Colloid Company, Arlington Heights, IL) was supplied by Mr. Micha Vaadia (Galil Mountain Winery). S9 "Pangel" sepiolite was purchased from Tolsa SA (Madrid, Spain). Wyoming Na-montmorillonite (SWy-2) was obtained from the Source Clays Repository of The Clay Minerals Society (Columbia, MO). Berberine chloride and egg-yolk L- $\alpha$ phosphatidylcholine (PC) were purchased from SigmaAldrich (Rehovot, Israel). Crystal violet (CV) was purchased as chloride salt from Fluka Chemie AG (Buchs, Switzerland). Mustard and Melaleuca essential oils were kindly supplied by Dr. Dan Gamrasni. All materials were used without further treatment or purification.

\subsection{Comparison with Gravimetric Measurements: Hygroscopic} Water Content on Clay. To mimic sorbents with different water content, KWK bentonite-berberine-clay with amounts ranging from 0.1 to 0.8 mmole berberine per g clay was prepared as described by Rytwo et al., 2005. At those added concentrations complete adsorption of the berberine is observed [18]. This was confirmed by measuring remaining berberine with an HP 8452A diode array UV-Vis spectrophotometer (Hewlett-Packard Co., Palo Alto, CA) at $344 \mathrm{~nm},(\varepsilon$ of berberine $22800 \mathrm{M}^{-1} \mathrm{~cm}^{-1}$ ). In all samples optical density (OD) was lower than the limit of detection (OD $<0.01$, equivalent to less than $4.4 \times 10^{-7} \mathrm{M}$ berberine remains). Organoclays were freeze-dried and left to equilibrate at ambient conditions for $72 \mathrm{~h}$. The water content was measured gravimetrically by weighing the air-dried and oven-dried (at $105^{\circ} \mathrm{C}$ for $24 \mathrm{~h}$ ) organoclay, respectively, on an analytical balance. Experiments were executed in triplicate.

2.3. Comparison with Mass Balance by UV-Vis Spectroscopy: Cationic Dyes Adsorption. Adsorption isotherms of crystal violet on SWy-2 montmorillonite were prepared and measured as described above for the berberine experiments. The concentration of the dye in each of the filtrates was determined by measuring the absorption at $588 \mathrm{~nm}$ ( $\varepsilon$ of $83000 \mathrm{M}^{-1} \mathrm{~cm}^{-1}$ ) using the HP 8452A spectrophotometer previously mentioned. The detection limit of the dye was $2.7 \times 10^{-7} \mathrm{M}$ corresponding to an optical density of 0.01 . Experiments were carried out in triplicate.

2.4. Comparison with CHNSO Measurements: Adsorption of Phosphatidylcholine. Phosphatidylcholine (PC) was adsorbed on clays (SWy-2, sepiolite) as liposomes from aqueous dispersions. Liposomes were prepared by the extrusion method; PC was dissolved in chloroform, which was subsequently evaporated under a stream of nitrogen. The dried lipid cake was rehydrated in $5 \mathrm{mM}$ $\mathrm{NaCl}$ solution at the desired concentration. The resultant suspension was repetitively extruded through polycarbonate membranes from Whatman with pore sizes of 400, 200, and $100 \mathrm{~nm}$, respectively. The final liposome diameter was ca. $130 \mathrm{~nm}$ as confirmed by dynamic light scattering measurements (Malvern Instruments Zetasizer Nano ZS). For adsorption on the clays, liposome dispersions of $0.1-$ $2.4 \mathrm{mM}$ PC concentration were contacted with the sorbates (at $2 \mathrm{mg} \mathrm{mL}^{-1}$ clay concentration) and stirred overnight. The clay-PC materials were recovered by centrifugation, washed, and subjected to ATR-IR and CHN elemental analysis (PerkinElmer 2400 Series II CHNS/O Elemental Analyzer), respectively. For quantitative PC analysis by ATRIR the PC/clay absorption intensity ratios were calibrated by collecting IR spectra of physical mixtures of clay with known amounts of PC.

2.5. Kinetic Evaluations: Essential Oils Evaporation from Clays and Organoclays. Measurements of essential oils were performed directly or by direct mixing with sorbents (SWy2 or organoclays based on CV or berberine adsorbed on SWy-2 at 0.8 mmole dye per g clay) at a $1: 10$ weight ratio oil: sorbent. ATR-FTIR measurements were performed as described below.

2.6. ATR-FTIR Measurements. Attenuated total reflectionFourier transform infrared (ATR-FTIR) spectra of all the experiments described above were measured on a Nicolet Avatar 320 FTIR (Nicolet Analytical Instruments, Madison, WI), using a MIRacle ATR device with a diamond crystal plate (Pike Technologies, Madison, WI). Spectra were recorded at $4 \mathrm{~cm}^{-1}$ nominal resolution with mathematical corrections yielding a $1.0 \mathrm{~cm}^{-1}$ actual resolution and 100 measurements were averaged. OMNIC 8.1 (Thermo Fisher Scientific Inc.) software analytical procedures were used to convert the spectra from ATR to absorbance and to perform additional analysis such as peak resolution. Quantification 


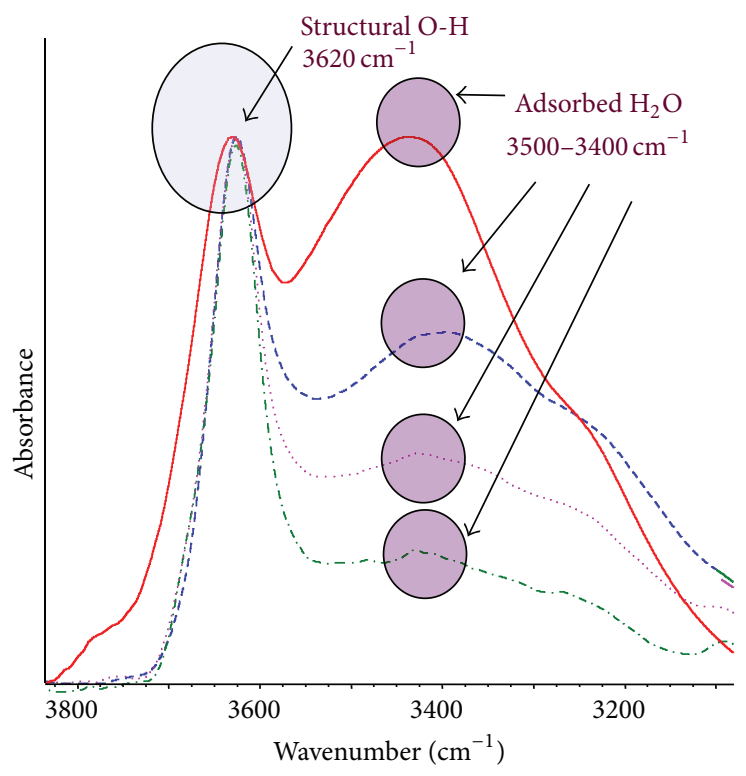

(a)

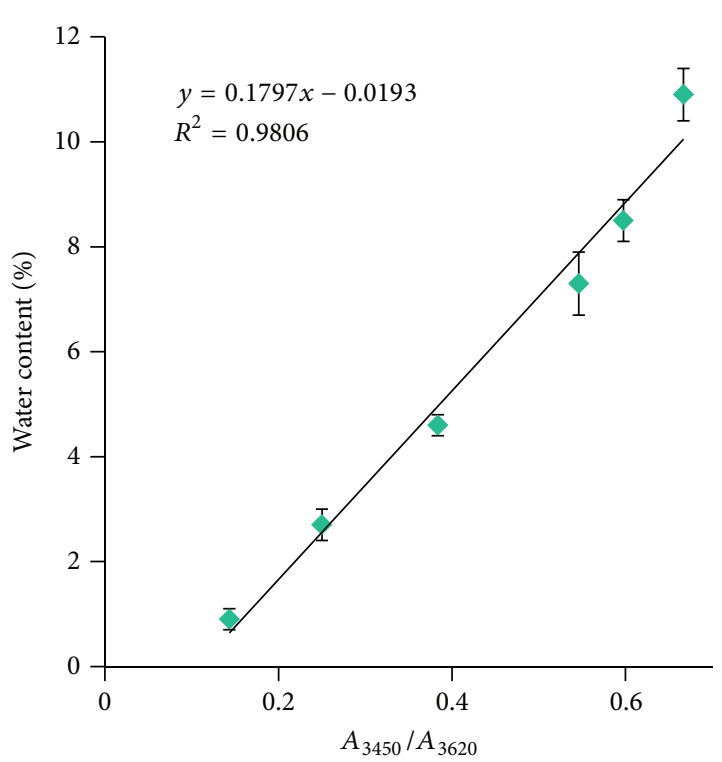

(b)

FIGURE 1: (a) FTIR spectrum of KWK raw clay (full line) and clay with 0.1 (dashed line), 0.25 (dotted line), and 0.75 (dashed dotted) mmole berberine per g KWK clay; (b) gravimetric water content of berberine-KWK organoclays and its relation to the ratio of IR absorption of adsorbed water $\left(A_{3450}\right)$ to structural $\mathrm{OH}\left(A_{3620}\right)$.

based on the absorption intensity of different peaks was performed using TQ analyst EZ 8.0.2.97 software (Thermo Fisher Scientific Inc.). Absorbance $(A)$ intensities for each relevant wavelength (e.g., $3450 \mathrm{~cm}^{-1}$ ) is denoted by $A_{3450}$. The quantitative evaluation of the amounts of adsorbed compound was based on the ratio between absorbance intensities at bands clearly ascribed to the adsorbate and the sorbate. This ratio is denoted, for example, by $A_{3450} / A_{3620}$ for adsorbed $\mathrm{O}-\mathrm{H}$ at $3450 \mathrm{~cm}^{-1}$ and structural $\mathrm{O}-\mathrm{H}$ at $3620 \mathrm{~cm}^{-1}$.

\section{Results}

3.1. Comparison with Gravimetric Measurements: Hygroscopic Water Content on Clay. The hygroscopic water content of clays decreases as the amount of adsorbed organic cation increases. The explanation of the effect is that the residual water content depends highly on the hydration shell of the adsorbed cations: exchanging inorganic hydrated cations by large organic molecules, with no hydration layers, leads to the formation of hydrophobic organoclay complexes, reducing the amount of hygroscopic water [19]. Adsorbed water on clay is observed at approximately $1600 \mathrm{~cm}^{-1}$ and a broad peak at approximately $3400 \mathrm{~cm}^{-1}$ corresponding to angle bending and stretching vibrations, respectively [20]. Vibrations due to $\mathrm{OH}$ moieties in the lattice structure of montmorillonite and bentonites exhibit a clear absorption band at approximately $3620 \mathrm{~cm}^{-1}[10]$. Figure 1(a) shows the FTIR spectra of raw KWK clay and clay with adsorbed berberine at different amounts, normalized to the latter peak.

A relative decrease of the adsorbed water stretching peak can be clearly observed. Similar results were presented for heated MX80 bentonite self-supporting films [20] and ascribed to a gradual decrease in water content due to the heating. In order to quantify this effect, we define the ratio $A_{3450} / A_{3620}$ as a quantitative indicator between the adsorbed water and structural $\mathrm{OH}$ groups. For example, when added berberine is 0.1 mmole berberine per g KWK clay, $A_{3450}=0.0653$ and $A_{3620}=0.1092$, yielding $A_{3450} / A_{3620}=$ 0.5977 . Figure 1(b) shows the relationship between this ratio and the hygroscopic water content of the berberine-KWK organoclays, as measured gravimetrically. It can be seen that a very good linear fit between both parameters is observed. Thus, by calibrating the measurement with a known water content, the ratio $A_{3450} / A_{3620}$ can be a good indicator for the water content of bentonite clays.

3.2. Comparison with Mass Balance by UV-Vis Spectroscopy: Cationic Dyes Adsorption. The most widely used evaluation technique of adsorbed amounts is based on mass balance calculations following measurement of the remaining concentration (not adsorbed) in solution. In most cases, when the adsorbate is an organic molecule with a large chromophore, the remaining concentration is measured by UV-Vis spectroscopy. As mentioned in the Introduction, this technique is prone to artifacts due to degradation, precipitation, or metachromatic changes in the spectrum of the adsorbate.

For the case of adsorption of crystal violet (CV) on bentonite clays, this technique is well established [21], even though degradation of the dye on vermiculite was reported [5]. Adsorption experiments of CV on SWy-2 montmorillonite were performed, with added dye between 0 and $1.6 \mathrm{mmole}^{-1}$, equivalent to $0-200 \%$ of the cation exchange capacity (CEC) of the clay. Adsorbed amounts were measured by mass balance as described in Section 2.3. 


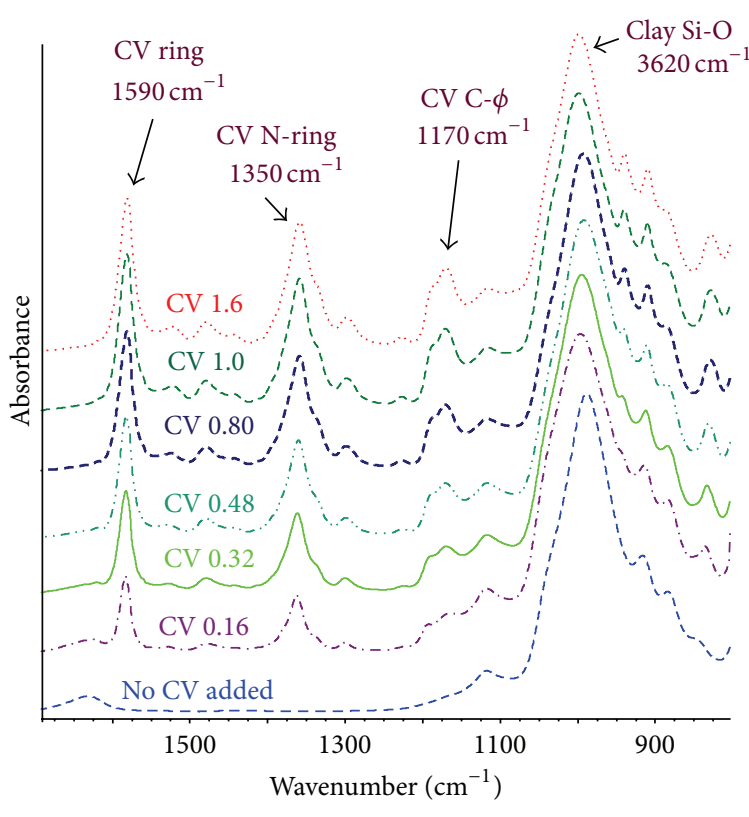

(a)

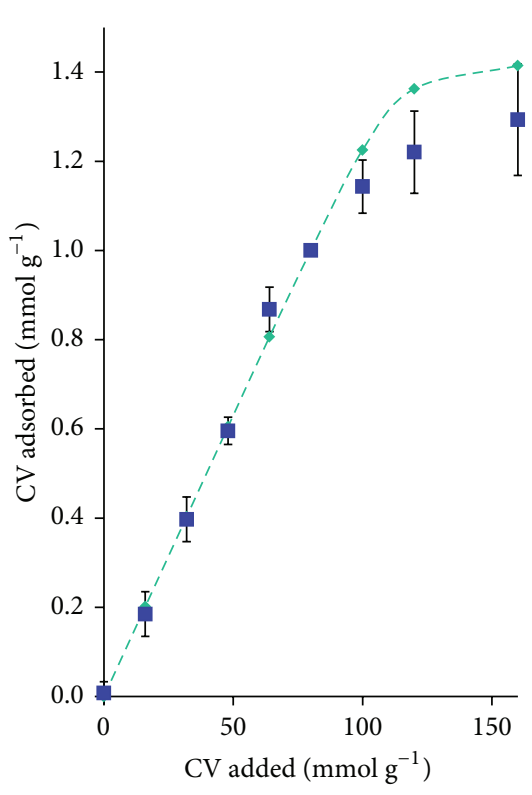

(b)

FIGURE 2: (a) ATR-FTIR spectra of SWy-2 raw clay and clay with added amounts of CV as denoted in the figure (in mmole $\mathrm{g}^{-1}$ clay). All spectra normalized by the absorption band of the Si-O vibration at $990 \mathrm{~cm}^{-1}$. (b) CV adsorbed as a function of the amount added. Points represent average adsorbed amounts evaluated by FTIR ratios of CV to clay absorption bands. Small dots and the dashed line represent the amount as measured by UV-Vis spectroscopy and mass balance calculation.

Figure 2(a) presents the ATR-FTIR spectra of the freezedried organoclay powder. The absorption band of the mineral ascribed to Si-O stretching [10] can be observed at about $1000 \mathrm{~cm}^{-1}$, whereas the additional bands observed in the spectra are ascribed [22] to moieties in triarylmethane dyes, as ring vibrations $\left(1590 \mathrm{~cm}^{-1}\right)$, C- $\phi$ stretching $\left(1170 \mathrm{~cm}^{-1}\right)$, and $\mathrm{N}$-ring vibrations $\left(1350 \mathrm{~cm}^{-1}\right)$. By using for calibration the data point of $100 \%$ CEC added CV, where all the dye is adsorbed [23], ratios between those bands and the clay SiO peaks $\left(A_{\mathrm{CV}, 1590} / A_{\mathrm{Si}-\mathrm{O}}, A_{\mathrm{CV}, 1350} / A_{\mathrm{Si}-\mathrm{O}}\right.$, and $\left.A_{\mathrm{CV}, 1170} / A_{\mathrm{Si}-\mathrm{O}}\right)$ were evaluated and related to the calibration data point. Results of the three ratios were averaged. Figure 2(b) exhibits the adsorbed amounts by ATR-FTIR evaluation compared with the values measured by UV-Vis and evaluated by mass balance. A relatively good fit can be observed $\left(R^{2}=0.9894\right)$ with slight discrepancies at large added concentrations, where the ATR-FTIR technique yields slightly lower (5$10 \%)$ adsorption values than the usual practice. A similar reasonable fit between the ATR-FTIR and the UV-Vis mass balance results was also measured for other cationic dyes (methylene blue, berberine, etc.) adsorbed on SWy-2 and other clays (KWK, S9 sepiolite, SHCa-1 hectorite, etc.).

\subsection{Comparison with CHNSO Measurements: Adsorption of} Phosphatidylcholine. Adsorption of phospholipids on clays is widely studied and used for several applications $[6,24]$. Since its absorption light in the UV-Vis range is only at $205 \mathrm{~nm}$, its quantification is usually determined by CHN measurements. Its vibrational spectrum has several specific absorption bands caused by the different functional groups:
$\mathrm{C}=\mathrm{O}$ stretching at $1720 \mathrm{~cm}^{-1-}, \mathrm{P}=\mathrm{O}$ asymmetric stretching bands at $1220-1240 \mathrm{~cm}^{-1}$ [25], methylene $\left(\mathrm{CH}_{2}\right)$ bending at $1440-1480 \mathrm{~cm}^{-1}$, and the $\mathrm{C}-\mathrm{H}$ stretching region at $2800-$ $3000 \mathrm{~cm}^{-1}$ [8]. All those bands can be used to quantify its adsorption, relative to significant bands of the sorbent as the Si-O band at approximately $1000 \mathrm{~cm}^{-1}$ or the $\mathrm{O}-\mathrm{H}$ vibrations in the lattice structure of the clay at approximately $3600 \mathrm{~cm}^{-1}[10]$. Figure 3 shows three of the four adsorbate bands, at several added amounts of phosphatidylcholine (PC) on SWy-2 montmorillonite, normalized to the sorbent as the $\mathrm{Si}-\mathrm{O}$ band at approximately $1000 \mathrm{~cm}^{-1}$. It can be seen that all PC peaks increase with the added amount, indicating increase in the adsorption.

Figure 4 shows the results of the adsorption of PC on SWy- 2 as evaluated by the average of ratios between four PC peaks $(\mathrm{C}=\mathrm{O}, \mathrm{CH}$ stretching, phosphate asymmetric vibration, and $\mathrm{CH}$ bend) and the $\mathrm{O}-\mathrm{H}$ vibrations in the lattice structure of the clay FTIR peaks, as a function of results measured by $\mathrm{CHN}$ analyzer for the same samples $\left(A_{1720} / A_{3620}, A_{2900} / A_{3620}\right.$, $A_{1230} / A_{3620}$, and $\left.A_{1460} / A_{3620}\right)$. The reason the O-H peak was preferred is that a PC peak at $1060 \mathrm{~cm}^{-1}$ might interfere in the accurate evaluation of the clay if the Si-O peak at $1100 \mathrm{~cm}^{-1}$ is used. A very good fit between the CHN and FTIR quantification is observed in Figure 4. Similar results were obtained for adsorption of PC on sepiolite clay.

3.4. Kinetic Evaluations: Essential Oils Evaporation from Clays and Organoclays. Essential oils are highly volatile substances isolated by a physical process from an odoriferous plant [26]. In recent decades essential oils and their components have 


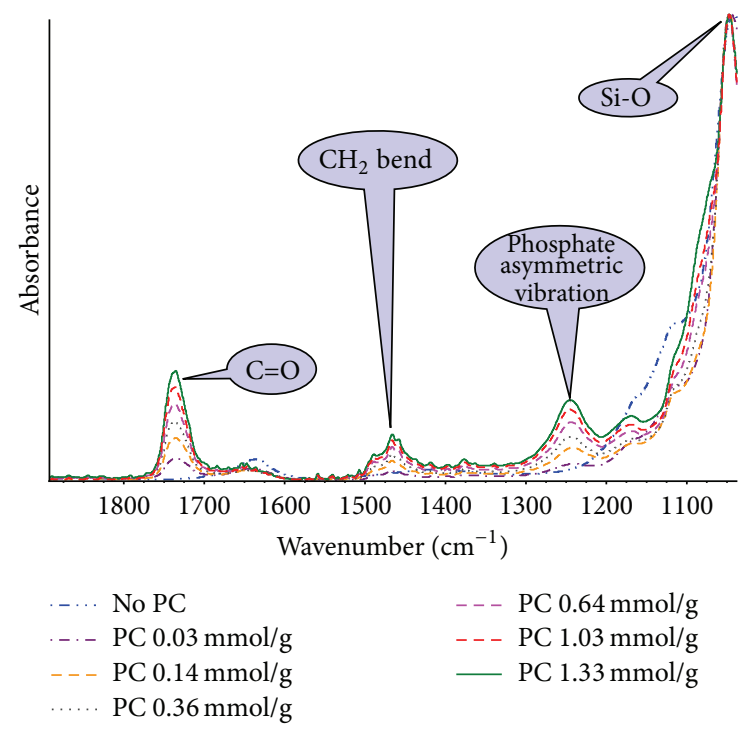

FIGURE 3: FTIR spectra of SWy-2 clay with added amounts of PC as denoted in the figure. All spectra normalized by the absorption band of the Si-O vibration at approximately $1000 \mathrm{~cm}^{-1}$.

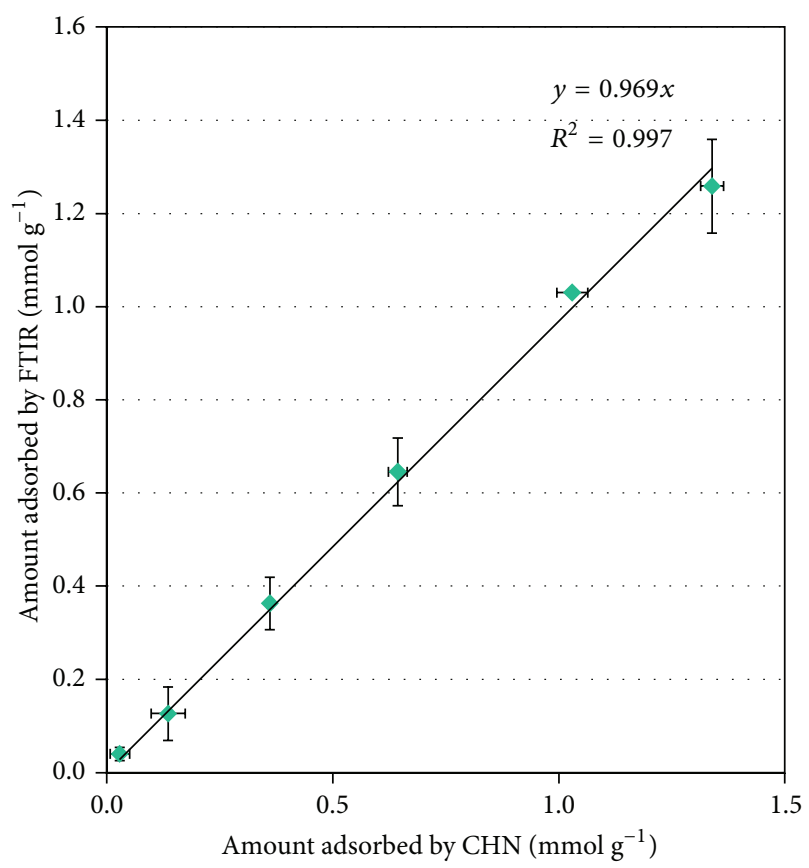

FIGURE 4: PC adsorbed on montmorillonite evaluated by FTIR ratios of PC to clay absorption bands, as a function of the measured adsorbed amounts by $\mathrm{CHN}$ analysis of the same samples.

been of great interest as they have been the sources of natural products [27]. Australian tea tree oil (TTO) is obtained from the foliage and terminal branches of species of Melaleuca, and its composition is given in the International Standard (ISO 4730-2004) [28]. Its main components are terpinen4-ol (30-48\%, CAS 562-74-3, and v.p. $0.0438 \mathrm{~mm} \mathrm{Hg}), \gamma$ terpinene (10-28\%, CAS 99-85-4, and v.p. $1.08 \mathrm{~mm} \mathrm{Hg}$ ), and $\alpha$-terpinene (5-13\%, CAS 99-86-5, and v.p. $1.64 \mathrm{~mm} \mathrm{Hg}$ ). As

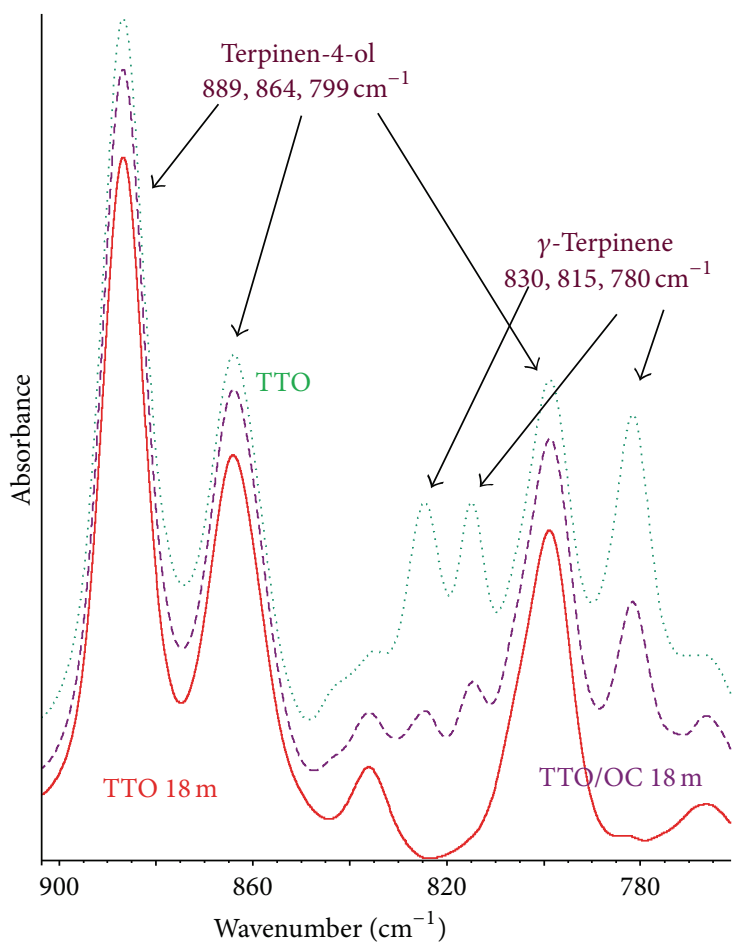

FIGURE 5: ATR-FTIR spectra of tea tree oil immediately after application (TTO) and after $18 \mathrm{~m}$ (TTO $18 \mathrm{~m}$ ), adsorbed on berberineSWy-2 organoclay after $18 \mathrm{~m}$ (TTO/OC $18 \mathrm{~m})$.

terpinenes are two orders of magnitude more volatile than terpinen-4-ol, the relative evaporation of those components might be monitored. In order to evaluate the contribution of the several compounds to the TTO mixtures, samples of the pure chemicals were purchased and measured. Pure $\gamma$ terpinene exhibits peaks at 780,815 , and $830 \mathrm{~cm}^{-1}$, whereas pure terpinen-4-ol presents absorption bands at 889,864 , and $799 \mathrm{~cm}^{-1}$. Figure 5 shows the FTIR spectrum of TTO immediately after application and after $18 \mathrm{~min}$ (TTO $18 \mathrm{~m}$ ). A relative decrease of the more volatile $\gamma$-terpinene compared to the less volatile terpinen-4-ol can be clearly observed. It is interesting to notice that the adsorption of TTO on a hydrophobic organoclay (berberine-SWy-2 montmorillonite) reduces terpinene evaporation, and its peaks remain substantial after $18 \mathrm{~min}$ (TTO/OC $18 \mathrm{~m}$ ).

By performing several measurements at different periods of time a detailed kinetic evaluation can be performed and by doing that the "slow release" effect obtained by the adsorption of the essential oil on the organoclay can be clearly observed. Figure 6 displays the natural logarithm of the ratio between the intensity of the $\gamma$-terpinene to the terpinen-4-ol absorption bands, as a function of the time from application. It can be seen that a reasonable fit to linear curves can be observed for all cases. A pseudo-first-order process behaves according to $d[A] / d t=-k[A]^{1}$, in which $[A]$ is the ratio between the peaks at a given time and $k$ is the kinetics coefficient of the process. Integration of this equation leads to $[A]=[\mathrm{C}] /[\mathrm{C}]_{0}=e^{-k t}$, which can be linearized to $\ln [A]=$ $-k t$. Thus, a linear representation of the logarithm of $[A]$ as 


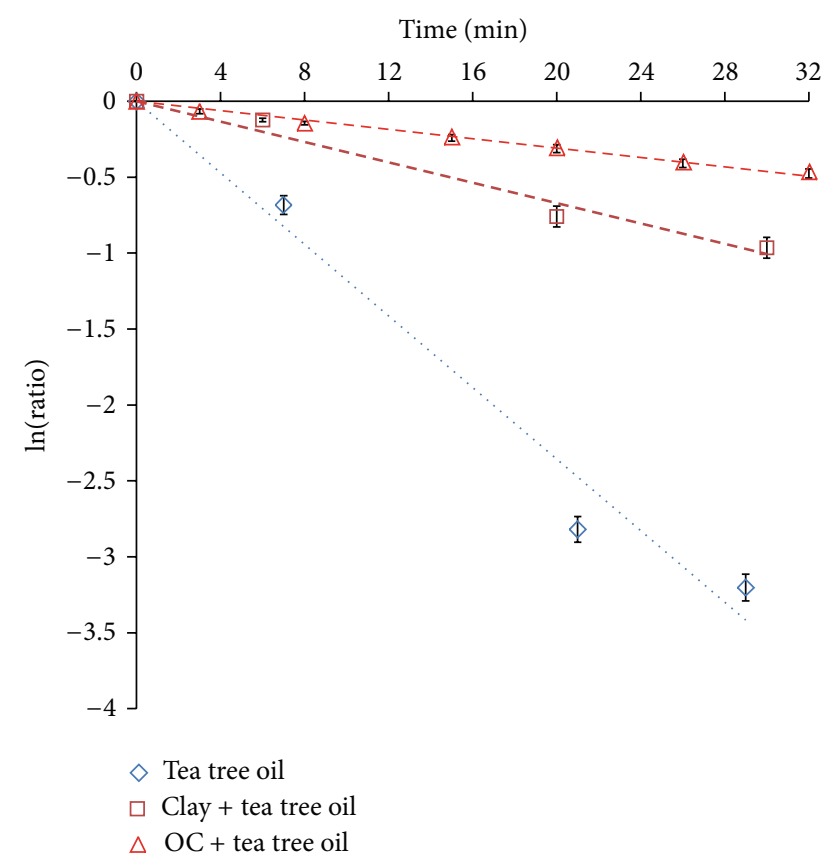

FIGURE 6: Natural logarithm of the ratio between the absorption bands of $\gamma$-terpinene to the terpinen-4-ol as a function of time for nonadsorbed tea tree oil, tea tree oil adsorbed on clay, and on berberine-montmorillonite (OC) organoclay.

a function of the time will yield the fit to a 1st-order process, and the slope will be the kinetic coefficient, which in this case represents the evaporation rate. Half-life can be calculated as $t_{1 / 2}=-\ln (1 / 2) / k=\ln 2 / k$ and is independent from the initial concentration [29].

Evaporation rate of $\gamma$-terpinene from tea tree yields a half-life time of $11.7 \mathrm{~min}$. Adsorbing the oil on raw clay or organoclay yields half-life time of 23.1 and $44.8 \mathrm{~min}$, respectively, demonstrating the slow release effect achieved by the hydrophobic berberine-SWy-2 hybrid platform.

An even more significant slow release effect is observed in mustard oil. The main component of this oil is allylisothiocyanate, which has a very distinctive IR absorption band at about $2100 \mathrm{~cm}^{-1}$. Figure 7 shows the evaporation of mustard oil as it can be seen from the almost complete disappearance of the isothiocyanate peak. The evaporation rate of the nonadsorbed oil is $0.193 \mathrm{~m}^{-1}\left(t_{1 / 2}=216 \mathrm{~s}\right)$. The adsorption of the oil on berberine-SWy-2 organoclay slows down the process by 40 folds, yielding an evaporation rate of $4.77 \times 10^{-3} \mathrm{~m}^{-1}\left(t_{1 / 2}=8720 \mathrm{~s}\right)$. Similar effects were measured with other essential oils and specific nanocomposites (Rytwo, 2014, unpublished results).

\section{Discussion}

Results presented in this study demonstrate a good fit between quantification based on ATR-FTIR measurements and other methods. Two main problems should be emphasized when using the proposed technique: (a) the quantitative determination of the technique proposed here depends on

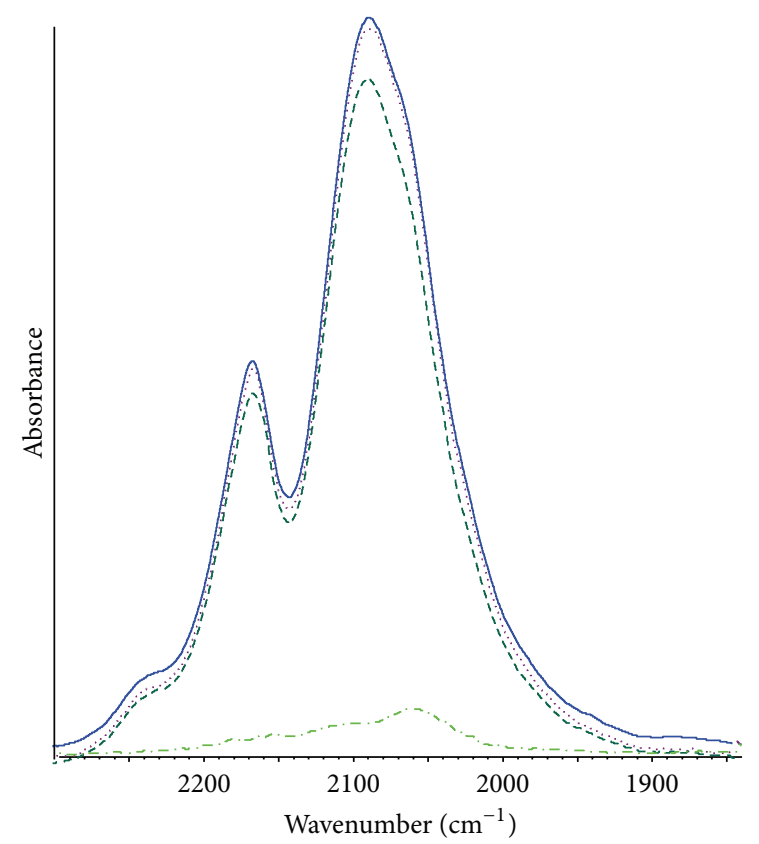

FIGURE 7: ATR-FTIR spectra of the isothiocyanate band of mustard oil immediately and $14 \mathrm{~m}$ after application (full and dashed dotted, resp.) compared to mustard oil adsorbed on berberine-SWy-2 organoclay immediately and $14 \mathrm{~m}$ after application (dotted and dashed lines, resp.).

the use of accurate calibration data; (b) there are several cases when adsorption of a compound causes changes in part of the FTIR absorption bands due to interactions with the adsorbent [18].

It is obvious that all measuring techniques are prone to biases and depend on accurate calibration. UV-Vis mass balance, for example, demands accurate determination of the $\varepsilon$ values of the analyte and the lack of interferences at the wavelength of measurement. Furthermore, it should be recalled that at high added concentrations usually considerable dilutions of the filtrate are needed, making mass balance subtractions more sensitive to errors. CHNSO and chromatography measurements rely also on calibrations performed sometimes on standards and matrices that differ completely from the analyte.

Thus, it is hard to determine which technique is more accurate, and each method should be used with caution and considering its limitations. However, considering the fact that differences between the presented methods hereby are minor and (for example) both UV-Vis mass balance and the proposed technique detected the saturation effect at high added concentrations of adsorbed organic dyes, the ATRFTIR method might be considered a possible alternative for fast quantification.

\section{Conclusions}

(i) ATR-FTIR is a fast, nondestructive, and relatively cheap technique that might allow direct semiquantitative measurements of components in mixtures, as 
in the case of adsorbed compounds, or of chemicals evaporating at different rates.

(ii) By using calibration samples, such measurements become quantitative.

(iii) The advantage of the technique is its lower susceptibility to over- or underestimations.

(iv) As any other analytical techniques, suitable preliminary tests are needed to ensure there are no significant changes in the intensity or the energy of the specific absorption bands used for the quantification.

\begin{tabular}{|c|c|}
\hline$A_{x x x}:$ & Absorbance at a wavelength of $x x x \mathrm{~cm}^{-1}$ \\
\hline ATR: & Attenuated total reflectance \\
\hline CEC: & Cation exchange capacity \\
\hline $\mathrm{CV}:$ & $\begin{array}{l}\text { Crystal violet [4-[bis[4-(dimethylamino) } \\
\text { phenyl]methylidene]cyclohexa-2,5-dien-1- } \\
\text { ylidene]-dimethylazanium }\end{array}$ \\
\hline FTIR: & Fourier transform infrared \\
\hline KWK: & Volclay KWK Food Grade bentonite clay \\
\hline OD: & Optical density \\
\hline PC: & Phosphatidylcholine \\
\hline SWy-2: & Wyoming Na-montmorillonite \\
\hline$t_{1 / 2}:$ & Half time life \\
\hline TTO: & Australian tea tree oil \\
\hline UV-Vis: & Ultraviolet-visible. \\
\hline
\end{tabular}

\section{Conflict of Interests}

The authors declare that there is no conflict of interests regarding the publication of this paper.

\section{Acknowledgments}

Bernd Wicklein thanks Comunidad de Madrid for financial support through Personal Investigador de Apoyo contract and E. Ruiz-Hitzky (ICMM-CSIC) for supporting this work through a CICYT project (Spain; MAT2009-09960).

\section{References}

[1] M. Borisover, E. R. Graber, F. Bercovich, and Z. Gerstl, "Suitability of dye-clay complexes for removal of non-ionic organic compounds from aqueous solutions," Chemosphere, vol. 44, no. 5, pp. 1033-1040, 2001.

[2] G. Rytwo, S. Nir, and L. Margulies, "Competitive adsorption to methylene blue and crystal violet to montmorillonite," Clay Minerals, vol. 28, no. 1, pp. 139-143, 1993.

[3] A. J. Aznar, B. Casal, E. Ruiz-Hitzky et al., "Adsorption of methylene blue on sepiolite gels: spectroscopic and rheological studies," Clay Minerals, vol. 27, no. 1, pp. 101-108, 1992.

[4] G. Rytwo, A. Banin, and S. Nir, "Exchange reactions in the CAMG-NA-montmorillonite system," Clays and Clay Minerals, vol. 44, no. 2, pp. 276-285, 1996.

[5] G. Rytwo, Y. Gonen, and R. Huterer-Shveky, "Evidence of degradation of triarylmethine dyes on texas vermiculite," Clays and Clay Minerals, vol. 57, no. 5, pp. 555-565, 2009.
[6] B. Wicklein, M. Darder, P. Aranda, and E. Ruiz-Hitzky, "Bioorganoclays based on phospholipids as immobilization hosts for biological species," Langmuir, vol. 26, no. 7, pp. 5217-5225, 2010.

[7] G. Rytwo, Y. Gonen, and S. Afuta, "Preparation of Berberinemontmorillonite-metolachlor formulations from hydrophobic/hydrophilic mixtures," Applied Clay Science, vol. 41, no. 1-2, pp. 47-60, 2008.

[8] J. Coates, "Interpretation of infrared spectra, a practical approach," in Encyclopedia of Analytical Chemistry, R. A. Meyers, Ed., pp. 10815-10837, John Wiley \& Sons, Chichester, UK, 2000.

[9] J. Madejová, "FTIR techniques in clay mineral studies," Vibrational Spectroscopy, vol. 31, no. 1, pp. 1-10, 2003.

[10] J. Madejová and P. Komadel, "Baseline studies of the clay minerals society source clays: infrared methods," Clays and Clay Minerals, vol. 49, no. 5, pp. 410-432, 2001.

[11] J. Bertaux, F. Fröhlich, and P. Ildefonse, "Multicomponent analysis of ftir spectra: quantification of amorphous and crystallized mineral phases in synthetic and natural sediments," Journal of Sedimentary Research, vol. 68, no. 3, pp. 440-447, 1998.

[12] S. Kaufhold, M. Hein, R. Dohrmann, and K. Ufer, "Quantification of the mineralogical composition of clays using FTIR spectroscopy," Vibrational Spectroscopy, vol. 59, pp. 29-39, 2012.

[13] C. E. Clarke, J. Aguilar-Carrillo, and A. N. Roychoudhury, "Quantification of drying induced acidity at the mineralwater interface using ATR-FTIR spectroscopy," Geochimica et Cosmochimica Acta, vol. 75, no. 17, pp. 4846-4856, 2011.

[14] C. E. Dowding, M. J. Borda, M. V. Fey, and D. L. Sparks, "A new method for gaining insight into the chemistry of drying mineral surfaces using ATR-FTIR," Journal of Colloid and Interface Science, vol. 292, no. 1, pp. 148-151, 2005.

[15] S. A. Boyd, G. Sheng, B. J. Teppen, and C. T. Johnston, "Mechanisms for the adsorption of substituted nitrobenzenes by smectite clays," Environmental Science and Technology, vol. 35, no. 21, pp. 4227-4234, 2001.

[16] J. M. Serratosa, "Infrared study of the orientation of chlorobenzene sorbed on pyridinium-montmorillonite," Clays and Clay Minerals, vol. 16, no. 1, pp. 93-97, 1968.

[17] G. Rytwo, S. Nir, and L. Margulies, "Interactions of monovalent organic cations with montmorillonite: adsorption studies and model calculations," Soil Science Society of America Journal, vol. 59, no. 2, pp. 554-564, 1995.

[18] G. Rytwo, Y. Gonen, S. Afuta, and S. Dultz, "Interactions of pendimethalin with organo-montmorillonite complexes," Applied Clay Science, vol. 28, no. 1-4, pp. 67-77, 2005.

[19] G. Rytwo, "Three simple experiments to demonstratethe impact of clay minerals on the behavior of organic pollutants in soils," in 2001. A Clay Odyssey: Proceedings of the 12th International Clay Conference, Bahia Blanca, Argentina, July 22-28, 2001, E. Dominguez, G. R. Mas, and F. Cravero, Eds., pp. 561-568, Elsevier Science B.V., Amsterdam, The Netherlands, 2003.

[20] J. Madejová, M. Janek, P. Komadel, H.-J. Herbert, and H. C. Moog, "FTIR analyses of water in MX-80 bentonite compacted from high salinary salt solution systems," Applied Clay Science, vol. 20, no. 6, pp. 255-271, 2002.

[21] J. Bujdak and N. Iyi, "Visible spectroscopy of cationic dyes in dispersions with reduced-charge montmorillonites," Clays and Clay Minerals, vol. 50, no. 4, pp. 446-454, 2002.

[22] L. Margulies and H. Rozen, "Adsorption of methyl green on montmorillonite," Journal of Molecular Structure, vol. 141, pp. 219-226, 1986, (Proceedings of the 17th European Congress on Molecular Spectroscopy). 
[23] G. Rytwo, R. Huterer-Harari, S. Dultz, and Y. Gonen, "Adsorption of fast green and erythrosin-B to montmorillonite modified with crystal violet," Journal of Thermal Analysis and Calorimetry, vol. 84, no. 1, pp. 225-231, 2006.

[24] B. Wicklein, M. Darder, P. Aranda, and E. Ruiz-Hitzky, "Phospholipid-sepiolite biomimetic interfaces for the immobilization of enzymes," ACS Applied Materials and Interfaces, vol. 3, no. 11, pp. 4339-4348, 2011.

[25] N. Toyran and F. Severcan, "Infrared spectroscopic studies on the dipalmitoyl phosphatidylcholine bilayer interactions with calcium phosphate: effect of vitamin $\mathrm{D}_{2}$," Spectroscopy, vol. 16, no. 3-4, pp. 399-408, 2002.

[26] E. B. Online, "essential oil," 2014, http://www.britannica.com/ EBchecked/topic/193135/essential-oil.

[27] W. Wang, N. Wu, Y. G. Zu, and Y. J. Fu, "Antioxidative activity of Rosmarinus officinalis L. essential oil compared to its main components," Food Chemistry, vol. 108, no. 3, pp. 1019-1022, 2008.

[28] E. Commission, SCCP Opinion on Tea Tree Oil, SCCP/1155/08, 2008, http://ec.europa.eu/health/ph_risk/committees/04_sccp/ docs/sccp_o_160.pdf.

[29] G. Rytwo and Y. Gonen, "Functionalized activated carbons for the removal of inorganic pollutants," Desalination and Water Treatment, vol. 11, no. 1-3, pp. 318-323, 2009, http://www.scopus .com/inward/record.url?eid=2-s2.0-77954240578\&partnerID= 40\&md5=6bbd4009453246bb9c78b0bd232df7bd. 

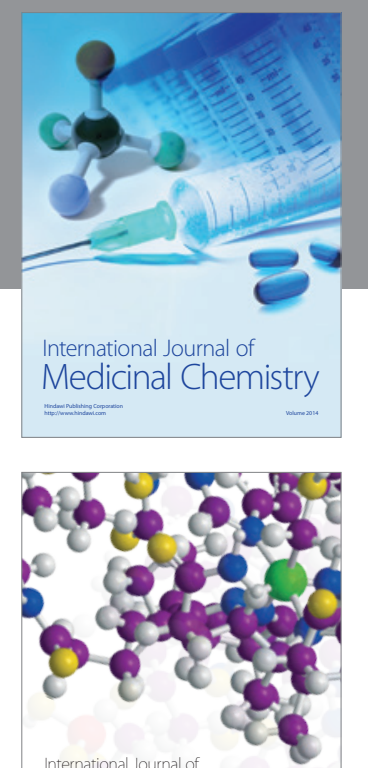

\section{Carbohydrate} Chemistry

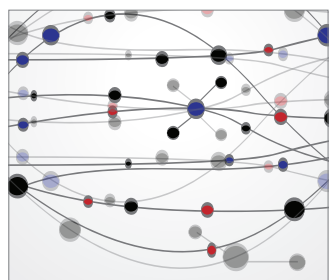

The Scientific World Journal
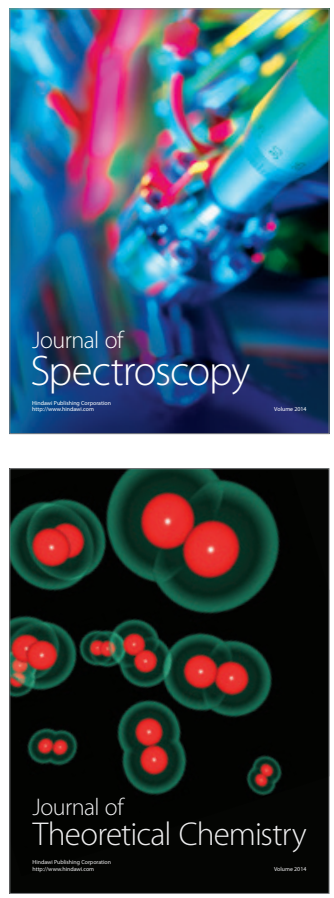
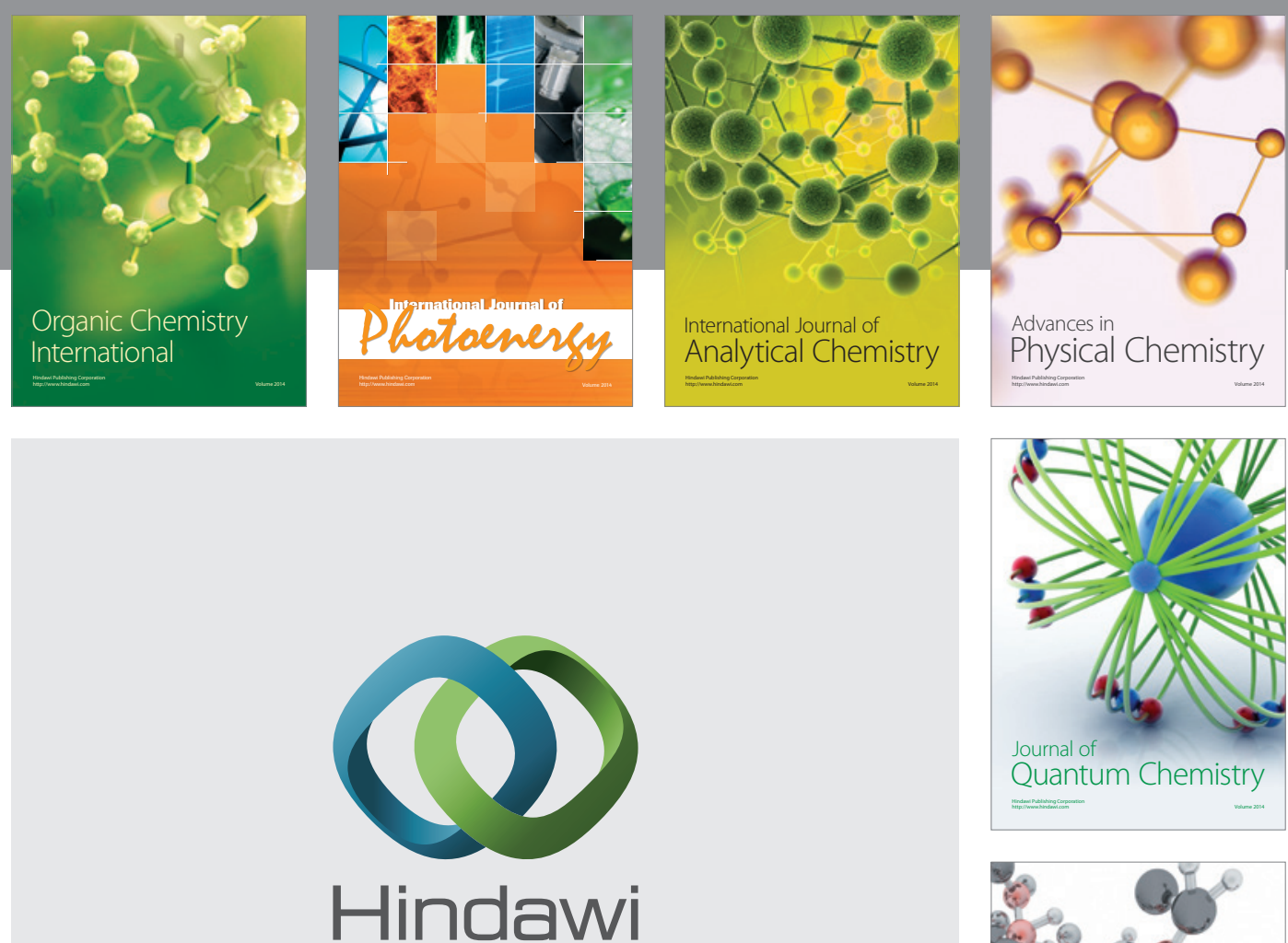

Submit your manuscripts at

http://www.hindawi.com

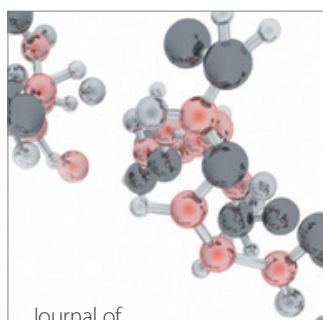

Analytical Methods

in Chemistry

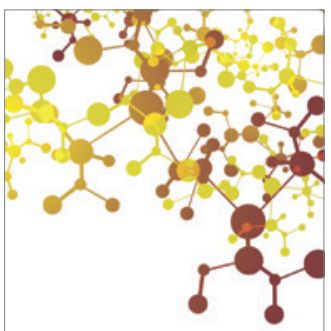

Journal of

Applied Chemistry

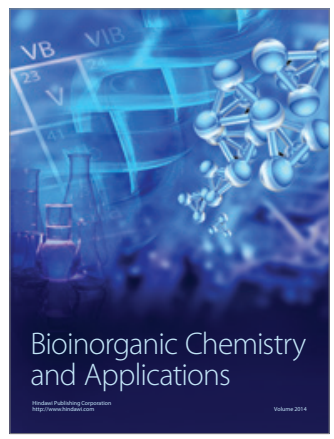

Inorganic Chemistry
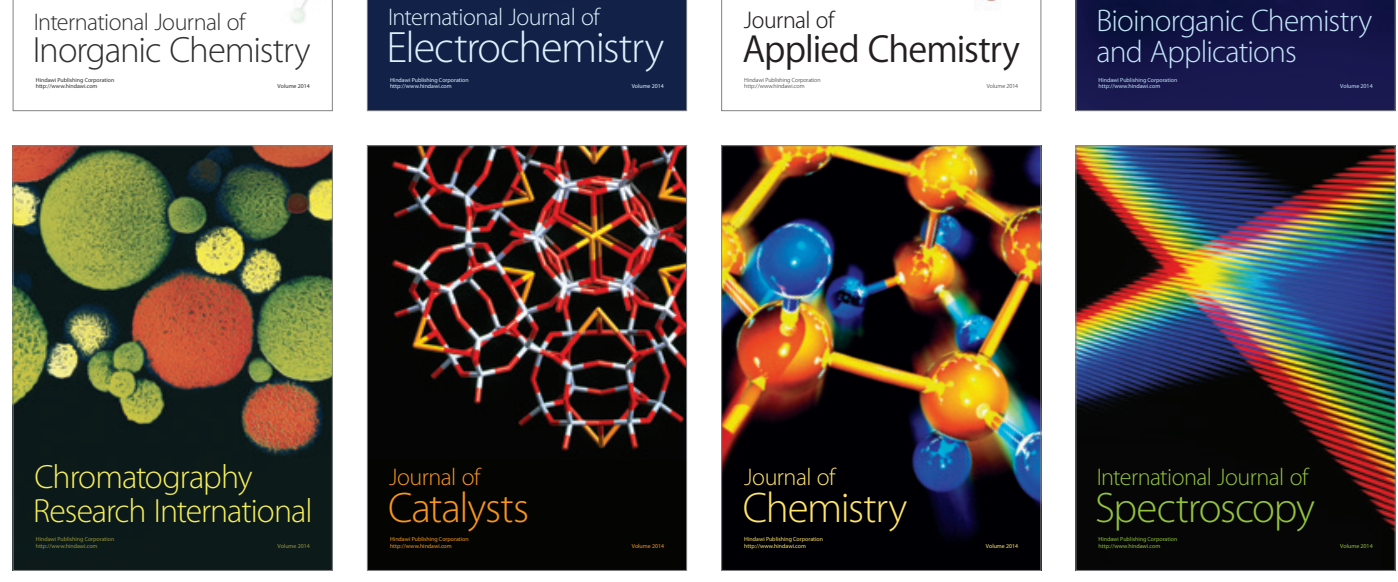